\title{
Is research into superconductivity still attractive?
}

In the years before Bednorz and Müller discovered "possible high- $T_{c}$ superconductivity" in the system $\mathrm{La}_{2-\mathrm{x}} \mathrm{Ba}_{\mathrm{x}} \mathrm{CuO}_{4},{ }^{1}$ superconductivity was considered settled and interesting more from a technical point of view. The situation in this subfield seemed comparable to that of general physics at the beginning of the twentieth century. The discovery of 1986 was in a sense the quantum leap of low-temperature condensed matter physics. Unprecedented activities were stimulated yielding a wealth of original results, even well beyond the cuprates, in materials science, theory, and experiment. However, the mechanism of superconductivity remaines elusive.

The problem of resolving this puzzle has its origin in the material class of the cuprates which have many instabilities in close proximity, and small differences in composition, purity or doping level have huge effects on the properties. In this situation, coordinated research is the only viable approach. Samples can be passed around and studied with various methods in order to disentangle generic and individual properties. At the time this joint activity has been started and supported by the German Research Foundation (DFG) the participating groups, both in theory and experiment, had an advanced arsenal of methods at hand which were very focussed and promising for the intended work. In many cases substantial progress had been achieved before. Therefore, the integration of the activities into a DFG Research Unit appeared to be a natural step forward.

In the two funding periods between 2003 and 2010 six and nine groups, respectively, in Aachen, Cologne, Dresden, Fribourg (Switzerland), Garching, Stuttgart, and Würzburg collaborated closely to gain insight into the intrinsic properties of the cuprates. Some of the latest results are compiled in this Special Topics issue of the European Physical Journal comprising three theoretical and eight experimental contributions.

In the following introductory article we briefly review the present status of the field of superconductivity. In spite of major progress, the core of the cuprates' enigma is still not unveiled while a completely unexpected family of iron-based high- $T_{c}$ superconductors ${ }^{2,3}$ not only posed new challenges towards a deeper understanding but also broadened the perspectives for systematic studies and for applications. Both classes, the cuprates and the iron-based materials, among various other exciting novel systems ${ }^{4,5}$ demonstrate that superconductivity remains a vibrant field.

Garching and Würzburg, August 2010 Rudi Hackl and Werner Hanke

\footnotetext{
1 J.G. Bednorz, K.A. Müller, Z. Phys. B 64, 189 (1986)

2 Y. Kamihara, T. Watanabe, M. Hirano, H. Hosono, J. Am. Chem. Soc. 130, 3296 (2008)

3 M. Rotter, M. Tegel, D. Johrendt, Phys. Rev. Lett. 101, 107006 (2008)

4 C. Pfleiderer, Rev. Mod. Phys. 81, 1551 (2009)

5 N. Toyota, M. Lang, J. Müller, Low-Dimensional Molecular Metals (Springer, Berlin-Heidelberg, 2007)
} 